\title{
Tipos funcionales de plantas según su respuesta a las perturbaciones en un bosque semideciduo micrófilo costero de Cuba Oriental
}

\author{
Functional types of plants in response to disturbance in a coastal \\ microphyll semi-deciduous forest of eastern Cuba
}

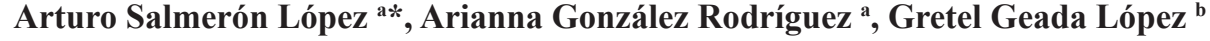 \\ *Autor de correspondencia: ${ }^{a}$ Museo de Historia Natural Tomás Romay, Centro Oriental de Ecosistemas y Biodiversidad, \\ Calle José A. Saco, N. 601. Santiago de Cuba. Cuba tel. +5322 626568, arturo@bioeco.cu \\ ${ }^{\text {b } U n i v e r s i d a d ~ d e ~ P i n a r ~ d e l ~ R i ́ o, ~ C e n t r o ~ d e ~ E s t u d i o s ~ F o r e s t a l e s, ~ C u b a . ~}$
}

\begin{abstract}
SUMMARY
As a consequence of human disturbance in the semi-deciduous forest in Siboney-Juticí Ecological Reserve, several changes have been produced. Futures actions for restoration in this vegetation require knowing the functional roll of the species during the postperturbations process. The disturbance level, considering its sources and characteristics, was assessed in some sites. Functional types were determined by the analysis of relative abundance in differently disturbed sites and the species functional traits (light demand or shade tolerance, fruits types and seed dispersion). With this information a cluster analysis was realized. Three functional types in response to disturbance were determined: spreaders, colonizers and stabilizer. Into each functional type, a group of species that defines the post disturbance dynamics and propitiates the restoration (dynamo-genetic species) was distinguished from another group of species (diversifiers) which confers diversity, redundancy and resilience to the system. The species redundancy within the functional types, although with differences in their abundances, allows them to be present and develop their functions in all disturbance levels. Because of this, the knowledge and use of the functional type constitute an important factor for developing ecological restoration strategies in this forest.
\end{abstract}

Key words: perturbation levels, dynamo-genetic species, redundancy, successional stages.

\section{RESUMEN}

Como consecuencia de las perturbaciones antrópicas producidas en el bosque semideciduo micrófilo de la Reserva Ecológica Siboney Juticí se produjeron cambios en la cobertura vegetal de sus ecotopos. Futuras acciones de restauración de las áreas antropizadas exigirían conocer el rol funcional de las especies durante los procesos postperturbación. En el trabajo se evaluó el nivel de las perturbaciones en diferentes sitios, considerando las fuentes y características de estas. Para la determinación de los tipos funcionales se analizaron la abundancia relativa de las especies en sitios desigualmente perturbados, los rasgos funcionales de estas (demanda de luz o tolerancia a la sombra, tipo de fruto, dispersión de las semillas) y se realizó un análisis de cluster. Se diferenciaron tres tipos funcionales de respuesta a las perturbaciones a los que se les denominaron: cobertoras, colonizadoras y estabilizadoras. Al interior de cada tipo funcional se diferenció un grupo de especies que condicionan la dinámica postperturbación y propician el avance de la recuperación (especies dinamogenéticas) mientras que otro grupo (diversificadoras) le confiere diversidad, redundancia y resiliencia al sistema. La redundancia de las especies dentro de los tipos funcionales definidos, aunque con diferencia en la composición y abundancia de las estas, les permite estar presentes y desempeñar su función en cualquiera de los niveles de perturbación, por lo que constituyen una poderosa herramienta para el desarrollo de estrategias de restauración ecológica en ese bosque.

Palabras clave: nivel de perturbación, especies dinamogenéticas, redundancia, estadios sucesionales.

\section{INTRODUCCIÓN}

Los tipos funcionales de plantas son conjuntos de especies que muestran respuestas similares al ambiente y efectos análogos en el funcionamiento de los ecosistemas (Díaz y Cabido 2001, Díaz et al. 2011) por lo que, para el caso de los bosques, son agrupaciones de plantas atendiendo al rol funcional de cada especie en determinados procesos eco- lógicos. Desde este punto de vista, la agrupación de estas especies tiende a estar basada más en atributos comunes que en relaciones filogenéticas. Los rasgos o características funcionales portados por las plantas son poderosos conductores de los procesos ecosistémicos. No existe una clasificación universal de tipos funcionales, la clasificación dependerá del objetivo del estudio, su escala y de los procesos ecológicos o ambientales de interés (Díaz y Cabido 2001). 
Los tipos funcionales de plantas han constituido una adecuada herramienta para el diagnóstico de procesos asociados a las perturbaciones (Venancio y Ribeiro 2002, Gondard et al. 2003) y para la elaboración y monitoreo a largo plazo de estrategias de manejo de ecosistemas y prácticas específicas de restauración (Gondard et al. 2003, Howort y Pendry 2006, Kooyman y Rossetto 2008). La redundancia funcional entre las especies, entendida como la capacidad común entre dos o más de ellas para el desarrollo de sus roles funcionales ha sido referida como una garantía de la resiliencia de los sistemas boscosos (Thompson et al. 2009, Petchey y Gaston 2009), particularmente en lo referido a la capacidad de estos sistemas para responder a perturbaciones de diferente índole.

La mayoría de los estudios consultados que analizan niveles de perturbación en la vegetación, realizan consideraciones cualitativas clasificando los sitios en no perturbados, moderadamente perturbados y perturbados (Mishra et al. 2004, Sahu et al. 2008 y Williams-Linera y Lorea 2009). Bhuyan et al. (2003) utilizan un índice dado por la relación porcentual entre el área basal de árboles cortados y el área basal del total de árboles. Jiménez (2012) considera como fuente de disturbio: tala selectiva, extracción de leña y productos forestales no maderables, claros por efecto del viento y caída de árboles, y afectación total por caminos, asignándole a cada una de estas variables un valor ordinal desde el caso sin disturbio (1) hasta disturbio alto (4).

Variaciones en la abundancia de las especies atribuibles a las perturbaciones antrópicas y a los procesos postperturbación han sido reportadas por varios autores (Lavorel 1999, Venancio y Ribeiro 2002, Gondard et al. 2003).

Según Matos (2006), existe un grupo de especies, a las que este autor denomina dinamogenéticas, las cuales constituyen la mayor parte de la vegetación, tienen la mayor cobertura y suelen alterar los equilibrios físicos haciendo avanzar la sucesión en dirección a los estadios climáxicos. Las especies dinamogenéticas pueden estar presentes en diferentes etapas sucesionales, correspondiendo a diferentes tipos funcionales de plantas y son ellas, en lo fundamental, las que condicionan la dinámica postperturbación en el sistema.

Aunque se ha llegado a considerar hasta 14 variables para la diferenciación (Delgado 2012 la mayoría de los trabajos dirigidos a la definición de tipos funcionales, no sobrepasan los cinco (Kooyman y Rossetto 2008, Makana y Thomas 2006, Howort y Pendry 2006, Gondard et al. 2003). De igual forma, aunque se ha llegado a definir hasta ocho tipos (Delgado 2012) la mayoría de los estudios no sobrepasan cuatro grupos funcionales (Makana y Thomas 2006, Howort y Pendry 2006).

Entre los rasgos utilizados para la determinación de tipos funcionales de respuesta a las perturbaciones se encuentran la abundancia en sitios desigualmente perturbados (Lavorel 1999, Venâncio y Ribeiro 2002, Gondard et al. 2003) la tolerancia a la sombra y la demanda de luz (Venâncio y Ribeiro 2002, Howort y Pendry 2006, Maka- na y Thomas 2006) la forma de dispersión de las semillas (Howort y Pendry 2006, Kooyman y Rossetto 2008) y el tipo de frutos (Kooyman y Rossetto 2008, Delgado 2012).

Lavorel (1999) establece que la dinámica postperturbación implica cambios en la abundancia de los "grupos de respuesta" a las perturbaciones. Venâncio y Ribeiro (2002) clasifican las especies arbustivas y arbóreas, según categorías sucesionales en pioneras, secundarias iniciales y secundarias tardías. Realizan además un análisis sobre la abundancia en función del tamaño del claro. Gondard et al. (2003) exponen una metodología de cinco pasos para la selección de grupos funcionales de respuesta que en general considera cambios en la composición de especies entre las diferentes formas de vida en respuesta a los niveles de perturbación.

Makana y Thomas (2006), en sus análisis, distinguen entre especies pioneras, demandantes de luz, tolerantes a la sombra y árboles y arbustos del sotobosque. Delgado (2012) considera que no todas las especies pioneras se encuentran siempre presentes en las perturbaciones que generan claros en la vegetación y que la presencia de especies pioneras, está relacionada con las dimensiones de los claros y el tipo de perturbación que ha tenido lugar.

Uriarte et al. (2010) exponen que la estructura de los rasgos funcionales en comunidades arbóreas difiere considerablemente entre grupos sucesionales de especies pioneras, secundarias y tardías y que la similitud de estos rasgos es mayor al interior de comunidades tardías sucesionalmente.

Al sur de Cuba Oriental, en una amplia faja de terrazas cársicas ubicadas en el tramo central de la costa, se encuentran bosques semideciduos micrófilos. Entre estos destacan los que se localizan en la Reserva Ecológica Siboney Juticí (González et al. 2013).

La constitución geológica de la reserva es fundamentalmente de calizas cuaternarias, fuertemente carsificadas y escalonadas hacia el Sur en terrazas marinas bien diferenciadas. Las precipitaciones no sobrepasan los $800 \mathrm{~mm}$ anuales. En las partes más altas de estas terrazas, así como en las depresiones donde se acumulan depósitos de arcillas resultantes de la decalsificación, se establecen bosques semideciduos micrófilos constituidos por Bursera simaruba (L.) Sargent., Colubrina elliptica (Sw.) Brizicky et W. L. Stern, Senna atomaria (L). H. S. Irwin et Barneby, Coccoloba diversifolia Jacq., Amyris elemifera L., Vachellia macracantha (Humb. et Bonpl. ex Willd.) Seigler et Ebinger, Picrodendrom baccatum (L.) Krug et Urb. ex Urb., Sideroxylon salicifolium (L.) Lam., etc., en el estrato arbóreo y Croton lucidus L., Eugenia sp., Gymnanthes lucida Sw., Oplonia sp., Erythroxylum rotundifolium Lunan, Tecoma stans (L.) Juss. ex Kunth, Exostema caribaeum (Jacq.) Roem. et Schult., Guetarda ellíptica Sw., Randia aculeata L., etc., en el estrato arbustivo (González et al. 2013).

Reyes y Acosta (2005) reportan nueve "tipos de hábitats" en la reserva, de los cuales dos corresponden explícitamente a ecotopos de bosque semideciduo micrófilo: el 
bosque semideciduo micrófilo propiamente dicho y el bosque arbustoso secundario en ecotopos de bosque semideciduo micrófilo. La vegetación potencial en un área donde se localiza el bosque arbustoso secundario con dominancia de Vachellia macracantha, otro de los nueve tipos de hábitats identificados en su estudio, corresponde a un bosque semidecíduo micrófilo ${ }^{1}$.

Figueredo et al. (2011) realizan la caracterización de los estadios sucesionales tempranos en un sitio antropizado en ecótopo de bosque semideciduo micrófilo en Juticí, identificando estadios sucesionales tempranos (fiera I y homeostasis I) al interior del mismo. En la fase fiera I observan mayor abundancia-dominancia de especies pioneras, heliófilas, y en la homeostasis I, las típicas del bosque semideciduo micrófilo, como parte de la regeneración natural. El estadio de homeostasis I constituye un equilibrio relativo, en el cual se ha establecido, en esta zona, un estrato arbóreo dominado por una especie heliófila de rápido crecimiento como Vachellia macracantha.

Dentro del territorio de la Reserva Ecológica, existen áreas correspondientes a ecotopos de bosques semideciduos micrófilos que, antes de su declaración como reserva, fueron sometidos a diferentes tipos de perturbaciones antrópicas, como talas rasas y selectivas con diferentes fines (producción de carbón, leña y estacas) cultivos, pastoreo

Dr. Orlando Joel Reyes Domínguez. Investigador Titular. Centro Oriental de Ecosistemas y Biodiversidad. Departamento de Botánica (joel@bioeco.cu). Comunicación personal. intensivo y ocasional, plantaciones forestales e invasión de especies exóticas. En sectores aislados dentro de la reserva se mantienen, ocasionalmente, algunos de estos disturbios. Como consecuencia de estas alteraciones se han producido cambios en la cobertura vegetal en los ecotopos correspondientes a bosques semideciduos micrófilos de la reserva, con la consiguiente pérdida de la integridad de los mismos.

Para trabajar en la restauración del bosque semideciduo micrófilo, es necesario conocer cuáles son las especies que más influyen en la recuperación del mismo y determinar cuál es el rol funcional de esas especies en este proceso. De acuerdo con los rasgos funcionales de las especies presentes en el bosque que definen su rol en la dinámica postdisturbios, tales especies pueden ser agrupadas en tipos funcionales de respuestas a las perturbaciones. El presente estudio tiene por objetivo determinar los roles funcionales de las especies en la dinámica postperturbación del bosque semideciduo micrófilo de la Reserva Ecológica Siboney Juticí, y agruparlas en tipos funcionales según su desempeño en el proceso de recuperación del bosque frente a los disturbios registrados en el mismo.

\section{MÉTODOS}

La Reserva Ecológica Siboney Juticí se localiza en el borde costero de la región oriental de Cuba, al sureste de Santiago de Cuba (figura 1). Posee una superficie total de 1.854 ha de los cuales, aproximadamente 1.050 ha son terrestres. Más del $70 \%$ de la superficie terrestre de

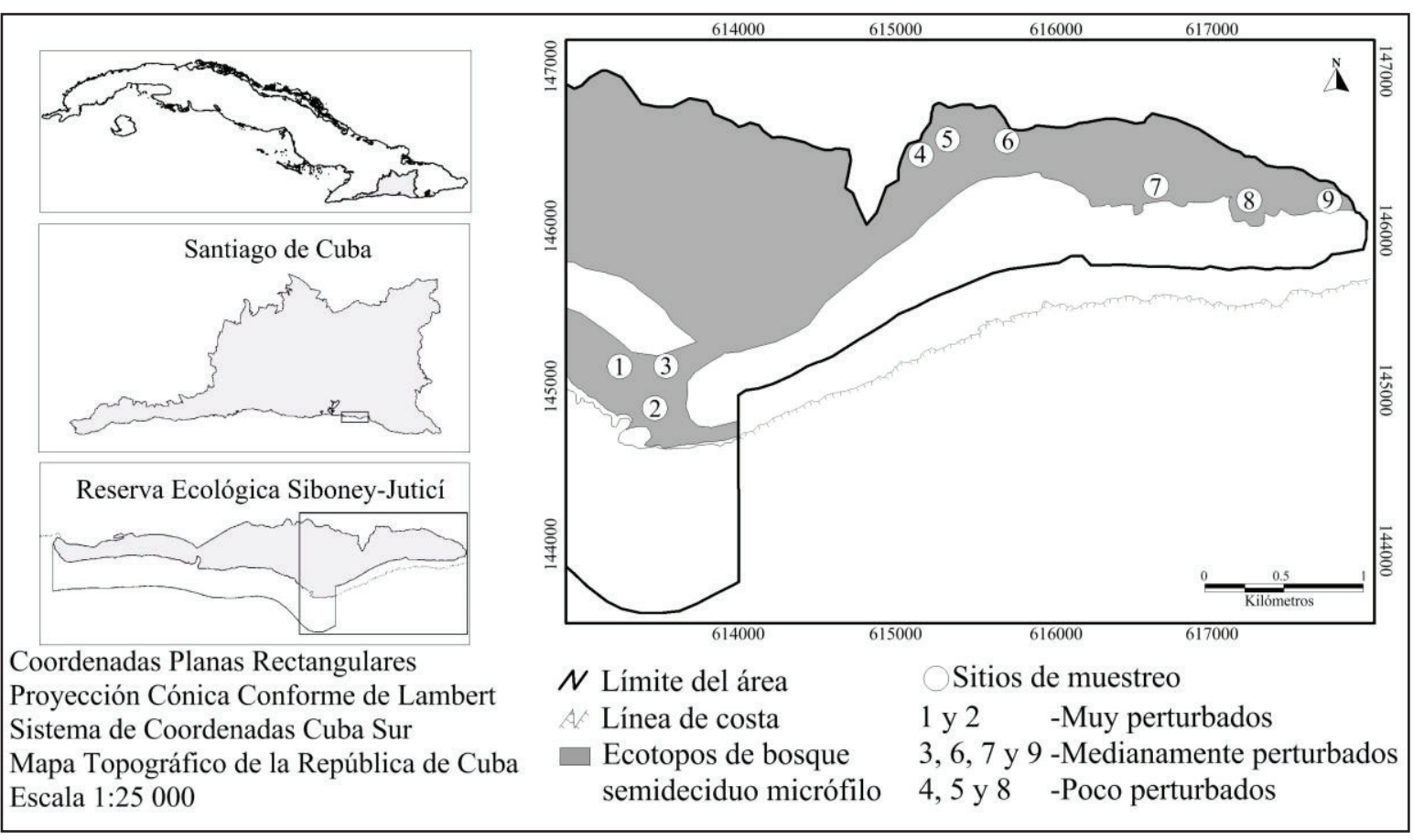

Figura 1. Ubicación de la Reserva Ecológica Siboney Juticí.

Location of Ecological Reserve Siboney Juticí. 
la Reserva Ecológica corresponde a ecotopos de bosques semideciduos micrófilos (González et al. 2013).

Niveles de perturbaciones en el bosque semideciduo micrófilo. Partiendo de los ecotopos referidos por Reyes y Acosta (2005), se evaluó el nivel de perturbación en los mismos, aplicando la siguiente ecuación:

$$
\mathrm{Pe}=\mathrm{Cu}+\mathrm{F}+\mathrm{Ca}+\mathrm{Ch}+\mathrm{Ei}+\mathrm{Pa}+\mathrm{T}
$$

Donde:

Pe: nivel de perturbación. De acuerdo con el valor de Pe, los sitios se consideraron como: poco perturbados $(0 \leq \mathrm{Pe}$ $\leq 3)$, medianamente perturbados $(4 \leq \mathrm{Pe} \leq 7)$, muy perturbados $(\mathrm{Pe}>7)$.

$\mathrm{Cu}$ : existencia de cultivos u otros movimientos de tierra (En últimos 5 años: 3 . En últimos 25 años: 2 . Más de 25 años atrás: 1 . No verificados en el sitio: 0 ).

F: fuego (En últimos 5 años: 3 . En últimos 25 años: 2. Más de 25 años atrás: 1 . Sin ocurrencia verificada: 0 ).

Ca: fabricación de carbón (En últimos 5 años: 3. En últimos 25 años: 2 . Más de 25 años atrás: 1 . Sin evidencias de fabricación de carbón: 0).

Ch: limpia de malezas o chapea(Actual: 3. Enúltimos 5 años: 2. En últimos 25 años: 1. No verificada en el sitio: 0 ).

Ei: presencia de especies invasoras (> $60 \%: 3.31 \%$ a $60 \%$ : 2.0 a $30 \%: 1$. No verificada en el sitio: 0 ).

Pa: pastoreo (Actual: 3. En últimos 5 años: 2. En últimos 25 años: 1. Sin verificar en el área: 0).

$\mathrm{T}$ : tala (Actual selectiva: 3. Rasa entre 20 y 30 años atrás o selectiva en los últimos cinco años: 2. Rasa más de 30 años atrás o selectiva en los últimos veinticinco años: 1 . No verificada en el sitio: 0).

Caracterización de los rasgos funcionales. En la investigación se tuvieron en cuenta solo las especies de arbustos y árboles leñosos que integran el bosque semideciduo micrófilo presente en la Reserva Ecológica Siboney Juticí, siguiendo la clasificación de formas de vida expuesta por Borhidi (1996) para las comunidades leñosas de Cuba. En el presente estudio se tuvieron en cuenta los siguientes: a) árboles gigantes (> $30 \mathrm{~m}$ ), megafanerófitos; b) árboles altos (15 a $30 \mathrm{~m}$ ) mesofanerófitos; c) árboles pequeños (5 a $15 \mathrm{~m}$ ) mesofanerófitos; d) árboles enanos y arbustos altos (2 a $5 \mathrm{~m}$ ) microfanerófitos; e) arbustos ( 0,5 a $2 \mathrm{~m}$ ) nanofaneróftios.

Para la determinación de la superficie mínima de la unidad de muestreo se aplicó la curva de acumulación de especies y se tuvo en cuenta el interés en capturar la información correspondiente a las especies dinamogenéticas (Matos 2006), por lo que se evaluó no solo la ocurrencia de las especies sino su abundancia relativa acumulada al incrementar la superficie de muestreo. Se consideraron las especies cuya abundancia relativa fuera igual o mayor que $0,5 \%$ en la superficie muestreada.

Debido a que las perturbaciones originan cambios en el número de especies, se aplicó un método similar al an- terior para determinar el tamaño de muestra según el nivel de perturbación.

Para la determinación de los tipos funcionales de respuesta a las perturbaciones se siguieron los criterios expuestos por otros autores (Lavorel 1999, Gondard et al. 2003, Kooyman y Rossetto 2008, Delgado 2012) adaptándolos a las condiciones y al objetivo del estudio, según se explica en los párrafos subsiguientes.

Abundancia de las especies presentes en sitios sometidos a diferentes niveles de perturbaciones antrópicas. Para el cálculo de la abundancia se inventariaron todos los individuos mayores de $1 \mathrm{~m}$ de altura. Los individuos de menos de $1 \mathrm{~m}$ de alto fueron contados en subparcelas de $1 \mathrm{~m}^{2}$ (dos por cada $25 \mathrm{~m}^{2}$ de superficie) uniformemente distribuidas dentro de la parcela, y a partir de esta medición se estimó el total de individuos menores de $1 \mathrm{~m}$ de cada especie para la parcela de $250 \mathrm{~m}^{2}$, considerando para esta la misma proporción (densidad superficial de individuos) que en el caso de las parcelas de $1 \mathrm{~m}^{2}$. El número total de individuos se tomó como la suma del total de individuos mayores de $1 \mathrm{~m}$ inventariados, más el número de individuos menores de 1 $\mathrm{m}$ estimados en la parcela.

Para cada especie se consideró la abundancia relativa en el tipo de sitio (poco, medianamente o muy perturbado), como la proporción que representa el número total de individuos de la especie respecto al número total de individuos de todas las especies presentes en todas las parcelas muestreadas para el sitio en cuestión. Debido a lo anterior, aunque algunas especies presentaron abundancia relativa mayor que $0,5 \%$ en la parcela de muestreo $\left(250 \mathrm{~m}^{2}\right)$, su abundancia relativa para el sitio (considerando el total de parcelas según el tamaño de muestra requerido para cada nivel de perturbación) pudo ser inferior a $0,5 \%$. En caso de que lo anterior ocurriera, las especies siguieron siendo consideradas para la determinación de los tipos funcionales.

Demanda de luz o tolerancia a la sombra de las especies. Para agrupar las especies presentes en demandantes de luz o tolerantes a la sombra, se establecieron en las parcelas bandas de $1 \mathrm{~m}$ de ancho equidistantes entre sí $(150 \mathrm{~m}$ en sitios muy perturbados, $300 \mathrm{~m}$ en medianamente perturbados y $250 \mathrm{~m}$ en los poco perturbados) que sumaron una longitud total de $700 \mathrm{~m}$ de largo por $1 \mathrm{~m}$ de ancho $\left(700 \mathrm{~m}^{2}\right)$.

En cada banda se midió el área total cubierta considerada como la suma de los tramos cubiertos por adultos de cualquier especie y el resto se consideró área descubierta. Para todas las especies considerando el estado regenerativo (plántula, juvenil o adulto), se contó el número de individuos y la superficie correspondiente a cada uno, asociada tanto a las áreas cubiertas como a las descubiertas.

Se determinó por especie, la cantidad de individuos juveniles o plántulas expuestos (asociado a las áreas descubiertas) y se evaluó la distribución Chi cuadrada (para comparar las proporciones entre el número de plántulas o juveniles cubiertos y expuestos, respecto de lo que cabría 
esperar teóricamente a partir de las proporciones entre el área total cubierta y el área total descubierta en cada banda. Aquellas para las que se encontró en áreas cubiertas un número significativamente mayor que el que cabría esperar fueron consideradas tolerantes a la sombra, en el caso contrario fueron consideradas demandantes de luz.

Tipo y tamaño de los frutos. Para la clasificación de los tipos y tamaños de los frutos se utilizaron las observaciones de campo y datos tomados de la versión digital de la Flora de Cuba que contiene todos los tomos publicados (CENPALAB 2000) y del resto de la bibliografía disponible (Borhidi 1996, Acevedo y Strong 2012). Según el tipo y tamaño de sus frutos, las especies se agruparon en cuatro categorías que se explican más adelante.

Forma de dispersión de las semillas. Para la clasificación de la forma de dispersión de las semillas se utilizaron las observaciones de campo, datos tomados de la bibliografía (CENPALAB 2000, Borhidi 1996, Reyes y González 2009), tratando de que la incertidumbre fuera mínima en este sentido. Las especies se agruparon según sus semillas fueran dispersadas por el viento, por animales o mediante autocoría.

Determinación de los tipos funcionales. Los tipos funcionales de plantas son considerados en este estudio como los grupos de respuesta a las perturbaciones que han tenido lugar en el bosque semideciduo micrófilo de la Reserva Ecológica. Es importante tener en cuenta el hecho de que dentro de un tipo funcional se encuentren varias especies (redundancia funcional) por la influencia reconocida (Thompson et al. 2009, Petchey y Gaston 2009) que ello tiene en la resiliencia del sistema. Para la determinación de los tipos funcionales se codificó la información obtenida sobre los rasgos funcionales considerados para cada una de las especies.

- Abundancia. Cuando la abundancia relativa de una especie fue $\geq 1 \%$ en algún sitio, se le dio el valor 3 en el sitio donde la abundancia relativa alcanza su mayor valor. Para la misma especie, si en alguno de los dos sitios restantes la abundancia relativa alcanzó un valor $\geq 60 \%$ del máximo ya reportado, se le asignó igualmente el valor de 3, de lo contrario, si solo alcanzó entre $30 \%$ y $59 \%$ o entre $1 \%$ y $29 \%$, se le asignaron valores de 2 o de 1, respectivamente. Si la especie no estuvo presente en alguno de los sitios, es decir la abundancia relativa fue cero entonces se le asignó directamente el valor 0 (cuadro 1). Cuando el máximo de la abundancia relativa de la especie en cualquiera de los tres sitios fue $<1 \% \mathrm{y} \geq 0,5 \%$, se le asignó a ese máximo el valor 2 , si en algunos de los sitios restantes mantuvo un valor $\geq 60 \%$ del máximo reportado, se le mantuvo el valor 2 , de lo contrario se le asignó el valor 1 o 0 si no estaba presente (cuadro 1). Si el máximo valor de la abundancia de la especie en algún sitio fue $<0,5 \%$, se le asignó el valor 1 y en el resto de los sitios solo tendría valores de 1 (presencia con abundancia relativa menor que $0,5 \%$ ) o 0 (ausencia de la especie en el sitio) (cuadro 1).

- Tolerancia a la sombra. La codificación según la tolerancia a la sombra fue: especies con tolerancia a la sombra verificada $=1$; especies con tolerancia a la sombra no verificada $=0$.

- Demanda de luz. La demanda de luz se codificó de la forma siguiente: especies con demanda de luz verifica$\mathrm{da}=1$; demanda de luz no verificada $=0$.

- Tipo de frutos. La codificación de las especies según los tipos de frutos fue la siguiente: legumbres, folículos y cápsulas con semillas aladas $=1$; cápsulas sin semillas aladas y frutos secos indehiscente $=2$; bayas $\mathrm{y}$ drupas con tamaño $<10 \mathrm{~mm}=3$; bayas y drupas con tamaño $\geq 10 \mathrm{~mm}$ tamaño $=4$.

- Forma de dispersión de las semillas. La codificación según la forma de dispersión de las semillas fue: especies con semillas anemócoras $=1$; especies con semillas autocoras $=2$; especies con semillas zoocoras $=3$.

Teniendo en cuenta que no se conoce a priori el número de tipos funcionales a definir en el bosque semideciduo micrófilo de la localidad de trabajo, se utiliza un análisis de cluster para agrupar a las especies evitando el sesgo puramente subjetivo, mediante el uso de las técnicas numéricas propias del método, y delimitar, de acuerdo a los resultados del análisis, un número de tipos funcionales coherente con el número de rasgos funcionales considerados y la factibilidad de su uso en los procesos de restauración que se lleven a cabo en la reserva.

Para el agrupamiento se empleó el software Statgraphic Centurion XVI.I. En el análisis se utilizó el método

Cuadro 1. Codificación de los valores de abundancia de las especies según sus rangos. Abmx: abundancia máxima en cualquiera de los sitios. Ab: abundancia en un sitio dado. Cod: codificación asignada en función del valor de abundancia $\mathrm{Ab}$.

Abundance values codification according to its functional traits: Abmx: maximum abundance in any site. Ab: abundance in a given site. Cod: assigned codification following the abundance value $\mathrm{Ab}$.

\begin{tabular}{llc}
\hline Abundancia relativa & & Cod \\
\hline & $\mathrm{Ab}=\mathrm{Abmx}$ & 3 \\
$\mathrm{Abmx} \geq 1 \%$ & $\mathrm{Ab} \geq 0,6(\mathrm{Abmx})$ & 3 \\
& $0,6(\mathrm{Abmx})<\mathrm{Ab} \geq 0,3(\mathrm{Abmx})$ & 2 \\
& $\mathrm{Ab}<0,3(\mathrm{Abmx})$ & 1 \\
\hline & $\mathrm{Ab}=\mathrm{Abmx}$ & 2 \\
$1 \%<\mathrm{Abmx} \geq 0,5 \%$ & $\mathrm{Ab} \geq 0,6(\mathrm{Abmx})$ & 2 \\
& $\mathrm{Ab}<0,6(\mathrm{Abm})$ & 1 \\
\hline $0 \%>\mathrm{Abmx}<0,5 \%$ & $\mathrm{Ab}=\mathrm{Abmx}$ & 1 \\
\hline $\mathrm{Abmx}=0$ & $\mathrm{Ab}=\mathrm{Abmx}=0$ & 0 \\
\hline
\end{tabular}


de Ward y para el cálculo de la distancia en el espacio definido por las variables, la distancia métrica City Block.

\section{RESULTADOS}

Niveles de perturbación. En los sitios correspondientes al hábitat de bosque semideciduo micrófilo (Reyes y Acosta 2005) solo se verificaron extracciones selectivas de árboles maderables, pero desde hace más de 40 años no se realizan actividades de este tipo. En algunos sectores se evidenció la utilización de madera para fabricación de carbón, realizada hace más de 25 años. Estas áreas se localizaron fundamentalmente en la zona de conservación estricta de la Reserva Ecológica aunque algunas estuvieron incluidas en la zona de uso público. Al aplicar la ecuación [1] para el cálculo del nivel de perturbación se verificó que estos sectores de la reserva corresponden a sitios poco perturbados, es decir $(0 \leq \mathrm{Pe} \leq 3)$.

En los territorios definidos por Reyes y Acosta (2005) como hábitat de bosque secundario en ecótopo de bosque semideciduo micrófilo se evidenciaron actividades de tala y fabricación de carbón hasta hace aproximadamente 40 años, así como el pastoreo de caprinos y ovinos. En la actualidad se mantiene el pastoreo de ovinos y caprinos de manera ocasional. Parte de esos territorios se localizaron en la zona de uso público de la reserva. Una parte de estos, que fue sometida a un nivel de extracción más intenso en los años 80, fue repoblada con Leucaena leucocephala y se ubicó en la zona de control de especies invasoras. Estas parcelas, luego de calcular el nivel de perturbación, se corresponden con sitios medianamente perturbados $(4 \leq \mathrm{Pe} \leq 7)$.

En los sectores ubicados en el hábitat de bosque arbustoso con predominancia de Vachellia macracantha (Reyes y Acosta 2005), a diferencia de los anteriores, se encontró al aplicar la ecuación [1] para el cálculo de Pe que algunos correspondían a sitios muy perturbados $(\mathrm{Pe}>7)$ mientras que otros se correspondieron con sitios medianamente perturbados $(4 \leq \mathrm{Pe} \leq 7)$.

En los primeros se comprobó que la vegetación fue devastada desde 1930 con el establecimiento de una vía férrea y un territorio de laboreo en función del propio ferrocarril, que posteriormente dejó de funcionar decayendo la actividad en esa zona. El área siguió siendo usada para el pastoreo, el establecimiento de cultivos y la fabricación da carbón. Desde el año 2000 el territorio fue incluido dentro de la Reserva Ecológica, como uno de los sectores de la zona de restauración, pero aún se mantiene el trasiego de personas, lo que ha propiciado la ocurrencia de incendios de mediana magnitud en parte de su territorio. De manera ilegal se mantiene la chapea para alimentación de ganado ubicado fuera del área y la extracción de leña. Estos sitios fueron evaluados como muy perturbados $(\mathrm{Pe}>7)$.

En otras áreas dentro de este tipo de cobertura, sin embargo, no se comprobó ni la chapea ni la extracción actual de leña. Estos sitios, al realizar el cálculo de Pe, calificaron como medianamente perturbados $(4 \leq \mathrm{Pe} \leq 7)$.
La aplicación del método de la curva de acumulación de especies (figura 2) arrojó que para especies con abundancia relativa igual o mayor que $0,5 \%$ la superficie mínima de muestreo puede ser de $250 \mathrm{~m}^{2}$

La determinación del tamaño de muestra según el nivel de perturbación arrojó cinco réplicas en sitios muy perturbados, 10 en sitios medianamente perturbados y siete en los poco perturbados a partir de la determinación del número mínimo de parcelas en cada sitio

Rasgos funcionales de las especies. Abundancia de las especies. Aunque varias de las especies estuvieron presentes en un solo sitio, una proporción elevada de estas se reportó en más de un nivel de perturbación, incluso en los tres niveles estudiados, pero con diferencias notables en la abundancia relativa según el nivel de perturbación del sitio (cuadro 2).

Demanda de luz y tolerancia a la sombra. Para la demanda de luz, las pruebas de Chi cuadrada resultaron significativas para las especies: Vachellia macracantha, Senna atomaria, Colubrina elliptica, Tecoma stans, Croton lucidus y Bursera simaruba (cuadro 2), mientras que en el caso de la tolerancia a la sombra esto ocurrió para las especies: Rauvolfia ligustrina, Randia aculeata, Erythroxylum havanense, Bourreria virgata, Adelia ricinella, Diospyros grisebachii, Eugenia montícola, Zanthoxylum pistaciifolium, Gymnanthes lucida, Heterosavia bahamensis, Coccoloba diversifolia, Amyris elemifera y Sideroxylon salicifolium (cuadro 2). El resto de las especies no pudieron ser definidas ni como demandantes de luz ni como tolerantes a la sombra (valor 0 en ambas columnas del cuadro 2) al no presentar Chi cuadrada significativa en ninguno de los casos.

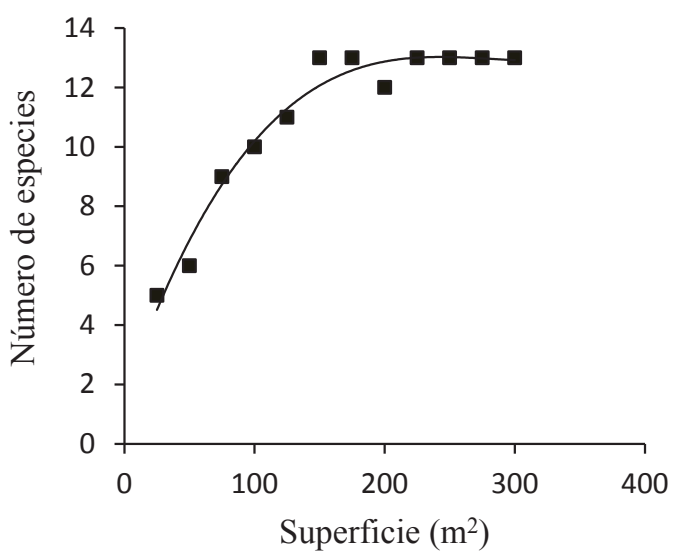

Figura 2. Curva de acumulación de especies con abundancia relativa $>0,5 \%$ según la superficie de muestreo en un bosque semideciduo poco perturbado de la Reserva Ecológica Siboney Juticí. La ecuación de la curva es $\mathrm{Y}=5,88 \mathrm{E}-07 \mathrm{x}^{3}$ $0,00048 \mathrm{x}^{2}+0,13 \mathrm{x}+1,49 . P<0,001$.

Accumulation curve from species with relative abundance $>$ $0.5 \%$ in relation to the sampling surface in a few perturbed microphyll semi-deciduous forest, in the Ecological Reserve Siboney Juticí. The equation of the curve is $\mathrm{Y}=5.88 \mathrm{E}-07 \mathrm{x}^{3}-0.00048 \mathrm{x}^{2}+0.13 \mathrm{x}+1.49 . P<0.001$. 
Cuadro 2. Rasgos funcionales de las especies estudiadas en sitios correspondientes a los niveles de perturbación considerados. Ab muy (med, poc) pert: abundancia relativa en los sitios muy (medianamente, poco) perturbados (\%). Fruto: tipo y tamaño de los frutos según la codificación expuesta en metodología. Semillas: forma de dispersión de las semillas según la codificación expuesta en métodos. Dmda de luz: si se verifica (1) o no (0) la demanda a la luz. Toler sombra: si se verifica (1) o no (0) la tolerancia a la sombra.

Functional traits of the studied species according to the different level of disturbance. Ab muy (med, poc) pert: relative abundance in high-level (middle-level, low-level) of disturbance (\%).Fruto: type and size of fruits related to the codification exposed in the methodology. Semilla: dispersion of seed related to the codification exposed in the methodology. Dmda de luz: if light demands are verified (1) or not (0). Toler sombra: if shade tolerance is verified (1) or not (0).

\begin{tabular}{|c|c|c|c|c|c|c|c|}
\hline Especies & $\begin{array}{c}\text { Ab muy } \\
\text { pert }\end{array}$ & $\begin{array}{c}\mathrm{Ab} \text { med } \\
\text { pert }\end{array}$ & $\begin{array}{c}\mathrm{Ab} \text { poc } \\
\text { pert }\end{array}$ & Fruto & Semilla & $\begin{array}{l}\text { Dmda } \\
\text { de luz }\end{array}$ & $\begin{array}{c}\text { Toler } \\
\text { sombra }\end{array}$ \\
\hline Vachellia macracantha (Humb. et Bonpl. ex Willd.) Seigler et Ebinger & 86,75 & 4,30 & 0,31 & 1 & 2 & 1 & 0 \\
\hline Senna atomaria $(\mathrm{L})$. H. S. Irwin et Barneby & 4,06 & 1,01 & 0,34 & 1 & 2 & 1 & 0 \\
\hline Rauvolfia ligustrina Willd. ex Roem. et Schult. & 0,51 & 0,00 & 0,00 & 3 & 3 & 0 & 1 \\
\hline Randia aculeata $\mathrm{L}$. & 3,91 & 31,47 & 5,09 & 3 & 3 & 0 & 1 \\
\hline Colubrina elliptica (Sw.) Brizicky et W. L. Stern & 0,00 & 8,16 & 3,51 & 2 & 2 & 1 & 0 \\
\hline Erythroxylum havanense Jacq. & 2,10 & 5,93 & 0,58 & 3 & 3 & 0 & 1 \\
\hline Bourreria virgata (Sw.) G. Don & 2,10 & 3,13 & 0,00 & 3 & 3 & 0 & 1 \\
\hline Erithalis fruticosa $\mathrm{L}$. & 0,00 & 2,71 & 1,65 & 3 & 3 & 0 & 0 \\
\hline Exostema caribaeum (Jacq.) Roem. et Schult. & 0,00 & 2,54 & 2,39 & 1 & 1 & 0 & 0 \\
\hline Adelia ricinella $\mathrm{L}$. & 0,00 & 2,32 & 0,00 & 2 & 3 & 0 & 1 \\
\hline Diospyros grisebachii (Hiern.) Standl. & 0,00 & 2,18 & 2,01 & 4 & 3 & 0 & 1 \\
\hline Eugenia monticola (Sw.) DC. & 0,00 & 1,87 & 0,07 & 3 & 3 & 0 & 1 \\
\hline Eugenia cowelli Britton et $\mathrm{P}$. Wilson & 0,00 & 1,29 & 0,18 & 3 & 3 & 0 & 0 \\
\hline Tecoma stans (L.) Juss. ex Kunth & 0,29 & 1,15 & 0,83 & 1 & 2 & 1 & 0 \\
\hline Melochia tomentosa $\mathrm{L}$. & 0,22 & 0,67 & 0,00 & 2 & 2 & 0 & 0 \\
\hline Zanthoxylum pistaciifolium Griseb. & 0,00 & 0,59 & 0,00 & 1 & 2 & 0 & 1 \\
\hline Guettarda cueroensis Britton & 0,00 & 0,59 & 0,00 & 3 & 3 & 0 & 0 \\
\hline Zanthoxylum fagara (L.) Sarg. & 0,00 & 0,45 & 0,00 & 1 & 2 & 0 & 0 \\
\hline Plumeria obtusa $\mathrm{L}$. & 0,00 & 0,42 & 0,20 & 1 & 1 & 0 & 0 \\
\hline Crossopetalum rhacoma Crantz & 0,00 & 0,42 & 0,18 & 3 & 3 & 0 & 0 \\
\hline Coulteria linnaei (Griseb.) Acev.-Rodr & 0,00 & 0,11 & 0,00 & 1 & 2 & 0 & 0 \\
\hline Pseudocarpidium ilicifolium (A. Rich.) Millsp. & 0,00 & 0,28 & 0,27 & 3 & 3 & 0 & 0 \\
\hline Cordia gerascanthus $\mathrm{L}$. & 0,00 & 0,08 & 0,00 & 3 & 3 & 0 & 0 \\
\hline Gymnanthes lucida Sw. & 0,00 & 2,29 & 22,06 & 2 & 2 & 0 & 1 \\
\hline Croton lucidus L. & 0,00 & 18,33 & 18,68 & 2 & 2 & 1 & 0 \\
\hline Heterosavia bahamensis (Britton) Petra Hoffm. & 0,00 & 0,00 & 14,79 & 2 & 2 & 0 & 1 \\
\hline Coccoloba diversifolia Jacq. & 0,00 & 3,07 & 11,93 & 2 & 2 & 0 & 1 \\
\hline Bursera simaruba (L.) Sargent. & 0,00 & 2,74 & 5,41 & 2 & 3 & 1 & 0 \\
\hline Guettarda elliptica $\mathrm{Sw}$. & 0,00 & 0,31 & 3,66 & 3 & 3 & 0 & 0 \\
\hline Amyris elemifera $\mathrm{L}$. & 0,00 & 0,31 & 1,72 & 3 & 3 & 0 & 1 \\
\hline Eugenia iteophylla Krug et Urb & 0,00 & 0,22 & 1,25 & 3 & 3 & 0 & 0 \\
\hline Sideroxylon salicifolium (L.) Lam. & 0,00 & 0,56 & 0,58 & 3 & 3 & 0 & 1 \\
\hline Picrodendron baccatum (L.) Krug et Urb. ex Urb. & 0,00 & 0,00 & 0,58 & 4 & 3 & 0 & 0 \\
\hline Pseudocarpidium avicennioides (A. Rich.) Millsp. & 0,00 & 0,22 & 0,51 & 3 & 3 & 0 & 0 \\
\hline Erythroxylum rotundifolium Lunan & 0,07 & 0,06 & 0,47 & 3 & 3 & 0 & 0 \\
\hline Thouinia trifoliata Poit. & 0,00 & 0,06 & 0,22 & 2 & 1 & 0 & 0 \\
\hline Banara minutiflora (A. Rich.) Sleumer & 0,00 & 0,00 & 0,16 & 3 & 3 & 0 & 0 \\
\hline Citharexylum spinosum $\mathrm{L}$. & 0,00 & 0,00 & 0,16 & 3 & 3 & 0 & 0 \\
\hline Tabebuia myrtifolia (Griseb.) Britton var. myrtifolia & 0,00 & 0,00 & 0,07 & 1 & 1 & 0 & 0 \\
\hline
\end{tabular}


Tipo y tamaño de los frutos. En nueve especies se comprobó la presencia de frutos en forma de legumbres, folículos o cápsulas con semillas aladas. En otras nueve se verificó la posesión de cápsulas sin semillas aladas o frutos secos indehiscentes. La mayoría, 19 especies, presentó bayas o drupas menores de $10 \mathrm{~mm}$, mientras que para solo dos especies se comprobó la presencia de bayas o drupas de $10 \mathrm{~mm}$ o más de tamaño (cuadro 2).

Forma de dispersión de las semillas. De acuerdo con la información disponible en la literatura y las observaciones de campo realizadas, las especies estudiadas se agruparon de la siguiente manera respecto a la forma de dispersión de la semilla (cuadro 2): semillas anemocoras: cinco especies; semillas autocoras: 11 especies; semillas zoocoras: 23 especies.

Tipos funcionales de respuesta a las perturbaciones. Una vez realizada la codificación de las especies según las características funcionales expuestas, el análisis de cluster para las mismas según esta codificación, arrojó el dendrograma que se muestra en la figura 3.

A partir del análisis del dendrograma obtenido mediante el análisis de cluster, se diferenciaron tres conglomerados de especies que se asocian a tipos funcionales y se les denominan: cobertoras, colonizadoras y estabilizadoras.

Especies cobertoras. En el primer conglomerado se encuentran las especies denominadas como cobertoras. En general son especies con predominancia en los sitios muy perturbados o medianamente perturbados. La mayoría son especies con frutos en forma de legumbre, folículos o cápsulas con semillas aladas o autocoras en ocasiones, demandantes de luz. Incluye las especies que invaden y cubren los claros en los ecotopos correspondientes a estos bosques generando una cobertura boscosa inicial de manera rápida. Este tipo funcional fue integrado por las siguientes especies: Vachellia macracantha, Croton lucidus, Colubrina elliptica, Bursera simaruba, Senna atomaria, Exostema caribaeum, Tecoma stans, Melochia tomentosa, Zanthoxylum fagara, Plumeria obtusa, Coulteria linnaei, Thouinia trifoliata y Tabebuia myrtifolia.

Especies colonizadoras. En el centro del dendrograma se distinguió un segundo grupo que se denominó colonizadoras. Este tipo está integrado en general por especies tolerantes a la sombra. Algunas poseen frutos en forma de cápsula con semillas autocoras y otras en forma de baya o drupa con semillas zoocoras. Este tipo funcional puede estar ampliamente distribuido en sitios correspondientes a casi todos los niveles de perturbación (cuadro 2). En general son especies con habilidad para, una vez que los claros hayan sido previamente ocupados por las cobertoras, establecerse debajo del dosel arbóreo de las primeras y colonizar espacios que ya tienen una cobertura boscosa previa. Este tipo funcional quedó integrado por las siguientes especies: Randia aculeata, Gymnanthes lucida, Heterosavia bahamensis, Coccoloba diversifolia, Erythroxylum havanense, Bourreria virgata, Adelia ricinella, Diospyros grisebachii, Eugenia monticola, Amyris elemifera, Zanthoxylum pistaciifolium, Sideroxylon salicifolium y Rauwolfia ligustrina,

Especies estabilizadoras. Al tercer grupo se le denominó estabilizadoras. En general presentaron frutos en forma de bayas y drupas, semillas identificadas como zoocoras o factibles para la zoocoría, y generalmente tolerantes a la sombra, aunque en algunos casos no fue verificada esta tolerancia. Estas especies estuvieron presentes tanto en sitios medianamente perturbados como en los sitios poco perturbados, pero generalmente con escasos individuos en el caso de estos bosques. Este tipo funcional estuvo integrado por las siguientes especies: Guettarda elliptica, Erithalis fruticosa, Eugenia cowelli, Eugenia iteophylla, Guettarda cueroensis, Picrodendron baccatum, Pseudocarpidium avicennioides, Erythroxylum rotundifolium, Crossopetalum rhacoma, Pseudocarpidium ilicifolium, Banara minutiflora, Citharexylum spinosum y Cordia gerascanthus.

\section{DISCUSIÓN}

En el estudio se prefiere utilizar un indicador cuantitativo para la evaluación de los niveles de perturbación debido a que permite distinguir entre áreas con disturbios similares. Tal es el caso de las diferenciadas al interior del bosque arbustoso secundario con dominancia de Vachellia macracantha, donde se encuentran sitios muy y medianamente perturbados. Un índice como el utilizado por Bhuyan et al. (2003) para estimar el nivel de perturbación, no es recomendable en este caso debido a que factores generadores de perturbación quedarían fuera del análisis.

Si se compara la composición y la abundancia de los sitios medianamente perturbados y poco perturbados (cuadro 2), se comprueba que estos sitios comparten un elevado número de especies y que la mayor diferencia entre estos radica en la abundancia relativa de ellas en cada sitio. Algunas especies son más abundantes en los sitios poco perturbados, mientras que otras lo son en los sitios medianamente perturbados. Se evidencia que el impacto de las perturbaciones es mayor sobre la abundancia que sobre la composición de especies, coincidiendo con lo obtenido por Lavorel (1999).

Aparentemente, lo anterior no se cumple estrictamente si se incrementa el nivel de las perturbaciones, de medianamente a muy perturbados, cuando el número de especies presentes, al menos en este caso y coincidente con varios autores (Bhuyan et al. 2003, Mishra et al. 2004, Sahu et al. 2008) disminuye drásticamente. Lo que sucede es que un fuerte incremento en el nivel de perturbación puede afectar drásticamente la abundancia de varias especies llevando el valor de la abundancia a cero. De hecho, en los sitios muy perturbados existe un pequeño número de especies, 


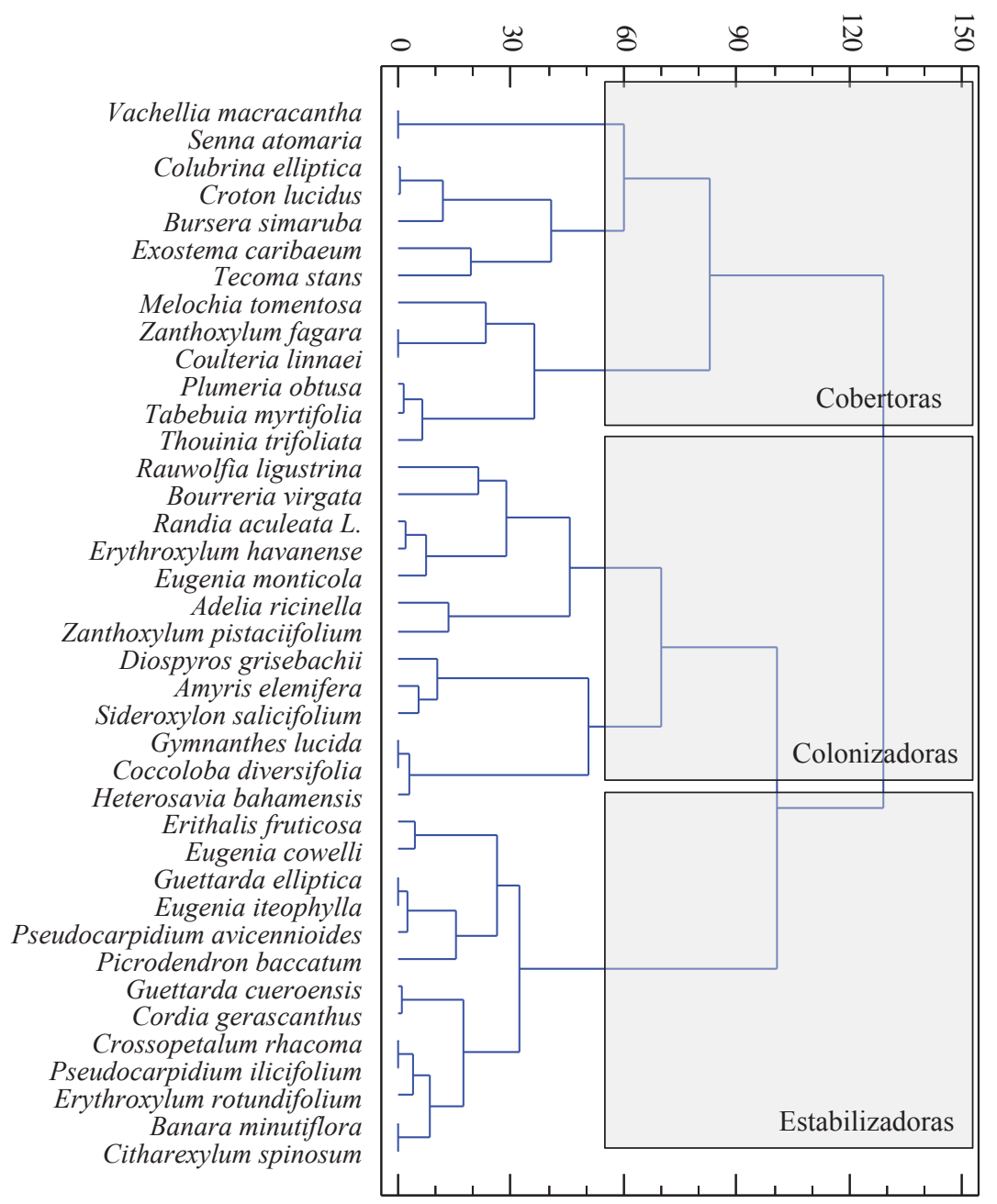

Figura 3. Dendrograma resultante del análisis de cluster para las especies reportadas según las características expuestas en el texto. Para una distancia de corte igual a 90 se identifican tres grupos de especies de acuerdo con los rasgos funcionales analizados.

Dendrogram resulting from the cluster analysis for reported species according to the characteristic exposed in the text. For a cut distance of 90, three species group are identified attending to the functional trait analyzed.

algunas compartidas con los otros dos sitios, y se mantienen diferencias significativas en sus abundancias relativas, sobre todo respecto a los poco perturbados.

Los resultados obtenidos refuerzan las afirmaciones sobre la importancia de conocer las variaciones en la abundancia de las especies para diferenciar los roles de estas en la dinámica de la respuesta postperturbación del ecosistema (Lavorel 1999, Venancio y Ribeiro 2002, Gondard et al. 2003) y aportan información en este sentido para el bosque semideciduo micrófilo de la Reserva Ecológica Siboney Juticí.

Las denominadas como especies cobertoras son siempre las que se establecen en los claros, aunque entre ellas puedan existir diferencias respecto a las dimensiones de los mismos. Ejemplos de diferencias en ese sentido han sido señaladas por Delgado (2012) para el bosque semideciduo de la península de Guanahacabibes. En la Reserva Ecológica Siboney Juticí, Vachellia macracantha y
Senna atomaria, tienden a ocupar los claros de mayores dimensiones, mientras que Colubrina elliptica y Bursera simaruba, suelen establecerse en claros de dimensiones medianas o pequeñas, aunque este aspecto no ha sido completamente probado en este estudio. Al parecer existen rasgos particulares que les pueden permitir desempeñar su rol funcional en diferentes tipos de perturbación. Estudios en este sentido deben ser realizados en el futuro.

De igual manera que en el caso de las cobertoras, las colonizadoras y las estabilizadoras son todas redundantes entre sí a los efectos de su rol funcional en el sentido analizado, sin embargo, existen diferencias entre ellas que pueden favorecer su establecimiento en circunstancias diferentes. Lo anterior tiene un vínculo indiscutible con la influencia reconocida de la redundancia funcional (presencia de varias especies dentro de un mismo tipo funcional) sobre la resiliencia del sistema a un espectro amplio de perturbaciones (Petchey y Gaston 2009, Thompson et al. 2009). 
En los tres tipos funcionales existen diferencias más o menos notables respecto a la abundancia de las especies que los integran. Si se consideran las especies más abundantes como aquellas que presentan abundancia relativa $>1 \%$ en cualquiera de los tres niveles de perturbación diferenciados, se tienen siete especies cobertoras, 10 de las especies señaladas como colonizadoras y solo cuatro de las especies estabilizadoras.

Las especies con abundancia relativa mayor que $1 \%$ (cuadro 2), se corresponden con las especies dinamogenéticas, según los criterios de Matos (2006). Estas especies pueden considerarse como las conductoras de los cambios en el ecosistema y son las que no deberían faltar en un proceso de restauración ecológica en los ecotopos de bosque semidecíduo micrófilo en la Reserva Ecológica Siboney Juticí. Un grupo de ellas son cobertoras, la mayoría son colonizadoras y algunas, las menos, estabilizadoras.

Las especies más escasas (abundancia relativa $<1 \%$ ), a las que se propone denominar como diversificadoras, incrementan la diversidad funcional del sistema, su redundancia y su resiliencia frente a las perturbaciones. La presencia de estas especies, fundamentalmente de las incluidas dentro del tipo de las estabilizadoras, puede conferirle mayor resiliencia a los estados más estables del sistema.

Si se tiene en cuenta que la clasificación en tipos funcionales ha considerado los rasgos funcionales de las especies y que la presencia de estos tipos funcionales juega un rol en la dinámica funcional del ecosistema, entonces estos resultados reafirman los planteamientos relacionados con el hecho de que los rasgos o características funcionales portados por las plantas son poderosos conductores de los procesos ecosistémicos (Díaz y Cabido 2001, Díaz et al. 2011) y que los tipos funcionales son una herramienta adecuada para el diagnóstico y planeamiento de procesos de restauración ecológica (Gondard et al. 2003, Howort y Pendry 2006, Koyman y Rossetto 2008).

A diferencia de otros autores que han enfocado más los estudios a la clasificación de las especies según su estadio sucesional, aunque utilizan rasgos funcionales para la diferenciación de las mismas, diferenciándolas luego en pioneras, secundarias y tardías (Venâncio y Ribeiro 2002, Howort y Pendry 2006) este estudio se enfoca en la clasificación funcional de las especies. Si bien existe una relación entre los rasgos funcionales de las especies y su frecuencia de aparición en estadios tempranos o tardíos de la sucesión vegetal, la clasificación propuesta, teniendo en cuenta que pretende diferenciar tipos funcionales, atiende más a la función de las especies en los procesos postperturbación, que a su diferenciación sucesional. De hecho, si se tiene en cuenta que los niveles de perturbación reflejan el tiempo post disturbio, estos resultados muestran que algunas especies correspondientes a un tipo funcional dado, pueden llegar a ser relativamente abundantes en más de un estadio postperturbación (cuadro 2), es decir, en más de un estadio sucesional.

La abundancia de algunas de las especies que se han definido como cobertoras en estadios sucesionales tempra- nos estudiados por Figueredo et al. (2011) apuntan a una confirmación de los resultados de Uriarte (2010) respecto a la diferenciación de rasgos funcionales entre especies correspondientes a diferentes estadios sucesionales.

No obstante, teniendo en cuenta los resultados expuestos y comparando con los de otros autores (Venâncio y Ribeiro 2002, Howort y Pendry 2006, Uriarte 2010, Figueredo et al. 2011) se puede afirmar que no todas las especies presentes en estados sucesionales tempranos tienen los rasgos de las denominadas como cobertoras en este estudio, como no todas las que están presentes en los estadios tardíos corresponden a especies estabilizadoras.

\section{CONCLUSIONES}

En el bosque semideciduo micrófilo de la Reserva Ecológica Siboney Juticí, se pueden definir tres tipos funcionales de plantas de acuerdo con el rol que estas desempeñan en la dinámica postperturbación: cobertoras, colonizadoras y estabilizadoras.

Una parte de las especies, en los tres tipos funcionales, son las que aceleran los cambios durante la recuperación actuando como dinamogenéticas y el resto actúa como especies diversificadoras.

Los tipos funcionales definidos pueden ser abundantes en más de un estadio sucesional, aunque no necesariamente con las mismas especies.

Las especies pertenecientes a los tipos funcionales descritos, particularmente las consideradas como dinamogenéticas, deben ser tenidas en cuenta en los procesos de restauración ecológica que se desarrollen en la reserva.

\section{REFERENCIAS}

Acevedo-Rodríguez P, MT Strong. 2012. Catalogue of Seed Plants of the West Indies. Washington D.C., USA. Smithsonian Institution Scholarly Press. $1221 \mathrm{p}$.

CENPALAB (Centro Nacional de Producción de Animales de Laboratorio, CU). Grupo de Investigaciones Especiales. 2000. Software Flora de Cuba, version 1.2 para Window 2000. La Habana, Cuba.

Bhuyan P, ML Khan, RS Tripathi. 2003. Tree diversity and population structure in undisturbed and human-impacted stands of tropical wet evergreen forest in Arunachal radesh, Eastern Himalayas, India. Biodiversity and Conservation 12: 1753-1773.

Borhidi A. 1996. Phytigeograohy and vegetation ecology of Cuba. Budapest, Hungría. Akademiai Kiado. 875 p.

Delgado F. 2012. Clasificación funcional del bosque semideciduo de la Reserva de la Biosfera Península de Guanahacabibes. Cuba. Tesis de Doctor en Ciencias. Pinar del Río, Cuba. Centro de Estudios Forestales. Universidad de Pinar del Río. 173 p.

Díaz S, F Quétier, DM Cáceres, SF Trainor, N Pérez-Harguindeguy, MS Bret-Harte, B Finegan, M Peña-Claros, L Poorter. 2011. Linking functional diversity and social actor strategies in a framework for interdisciplinary analysis of nature's benefits to society. PNAS 108(3): 895-90. 
Díaz S, M Cabido. 2001. Vive la différence: plant functional diversity matters to ecosystem processes. Trends in Ecology \& Evolution 16(11): 646-655.

Figueredo LM, RN Ramírez, F Acosta. 2001. Estudios sucesionales en un sitio antropizado en ecótopo de bosque semideciduo micrófilo en Juticí, Santiago de Cuba. Foresta Veracruzana 13(1):15-22.

Gondard H, S Jauffret, J Aronson, S Lavorel. 2003. Plant functional types: a promising tool for management and restoration of degraded lands. Applied Vegetation Science 6: 223-234.

González A, M Abad, A Salmerón, LO Alvarez, M Silot. 2013. Plan de Manejo de la Reserva Ecológica Siboney Juticí. La Habana, Cuba. Centro Oriental de Ecosistemas y Biodiversidad. $250 \mathrm{p}$.

Howorth RT, CA Pendry. 2006. Post-cultivation secondary succession in a Venezuelan lower montane rain forest. Biodiversity and Conservation 15:693-715.

Jiménez J A. 2012. Contribución a la ecología del bosque semideciduo mesófilo en el sector oeste de la Reserva de la Biosfera "Sierra del Rosario", orientada a su conservación. Tesis de Doctor en Ciencias Forestales. Pinar del Río, Cuba. Centro de Estudios Forestales, Universidad de Pinar del Río. 160 p.

Kooyman R, M Rossetto. 2008. Definition of plant functional groups for informing implementation scenarios in resourcelimited multi-species recovery planning. Biodiversity Conservation 17:2917-2937.

Lavorel S. 1999. Ecological diversity and resilience of Mediterranean vegetation to disturbance. Diversity and Distributions 5: 3-13.

Makana J R y S C Thomas. 2006. Impacts of selective logging and agricultural clearing on forest structure, floristic composition and diversity, and timber tree regeneration in the Ituri Forest, Democratic Republic of Congo. Biodiversity and Conservation 15:1375-1397.

Matos J. 2006. Manual de Manejo de Flora Silvestre. Universidad Martha Abreu de Las Villas. Villa Clara, Cuba. Edito- rial Feijoo. 247 p.

Mishra BP, OP Tripathi, RS Tripathi, HN Pandey. 2004. Biodiversity and Conservation 13: 421-436.

Petchey OL, KJ Gaston. 2009. Effects on ecosystem resilience of biodiversity, extinctions, and the structure of regional species. Theoretical Ecology 2:177-187.

Reyes J, F Acosta. 2005. Vegetación Terrestre. In Fong A, D Maceira, WS Alverson, JM Shopland eds. Cuba: SiboneyJuticí. Rapid Biological Inventories, Report 10. Chicago, USA. The Field Museum. p. 46-50.

Sahu P K, R Sagar, JS Singh. 2008. Tropical forest structure and diversity in relation to altitude and disturbance in a Biosphere Reserve in central India. Applied Vegetation Science 11: 461-470.

Thompson I, B Mackey, S McNulty, A Mosseler. 2009. Forest Resilience, Biodiversity, and Climate Change. A synthesis of the biodiversity/resilience/stability relationship in forest ecosystems. CBD Technical Series 43. Ontario, Canada. Secretariat of the Convention on Biological Diversity. 67 p.

Uriarte M, NG Swenson, RL Chazdon, LS Comita, WJ Kress, D Erickson, J Forero-Montana, JK Zimmerman, J Thompson. 2010. Trait similarity, shared ancestry and the structure of neighbourhood interactions in a subtropical wet forest: implications for community assembly. Ecology Letters 13: 1503-1514.

Reyes A, PA González. 2009. Dieta de Cyclura nubila nubila (Reptilia: Iguanidae) en la Reserva Ecológica Siboney Juticí, Santiago de Cuba. Mesoamericana 13(3): 34-40.

Venancio S, R Ribeiro. 2002. Gap-phase regeneration in a semideciduous mesophytic forest, south-eastern Brazil. Plant Ecology 00: 1-12.

Williams-Linera G, F Lorea. 2009. Tree species diversity driven by environmental and anthropogenic factors in tropical dry forest fragments of central Veracruz, Mexico. Biodiversity and Conservation 18(12): 3269-3293. DOI: 10.1007/ s10531-009-9641-3. 
